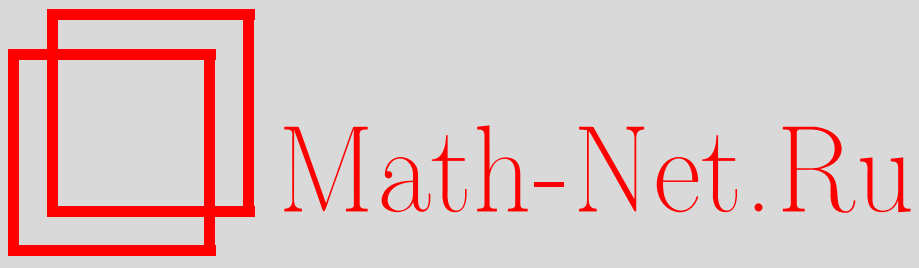

Н. Н. Пустовойтов, Оценка наилучших приближений периодических функций тригонометрическими полиномами через усредненные разности и многомерная теорема Джексона, Матем. сб., 1997, том 188, номер 10, 95-108

DOI: https://doi.org/10.4213/sm265

Использование Общероссийского математического портала Math-Net.Ru подразумевает, что вы прочитали и согласны с пользовательским соглашением

http://www. mathnet.ru/rus/agreement

Параметры загрузки:

IP : 54.224 .60 .19

26 апреля 2023 г., $16: 40: 45$ 
УДК 517.5

\author{
Н.Н. Пустовойтов
}

\title{
Оценка наилучших приближений периодических функций тригонометрическими полиномами через усредненные разности и многомерная теорема Джексона
}

В первом параграфе данной работы изучаются наилучшие приближения периодических функций одной вещественной переменной тригонометрическими полиномами. Указанные приближения оцениваются через усредненные разности. Во втором параграффе дается многомерное обобщение оценок из первого раздела. Как следствие этого результата получается многомерная теорема Джексона.

Библиограбоя: 7 названий.

\section{$\S 1$.}

Будем рассматривать $2 \pi$-периодические функции одной вещественной переменной $f(x)$. Считаем, что

$$
\begin{aligned}
\|f\|_{p} & =\left(\frac{1}{2 \pi} \int_{0}^{2 \pi}|f(x)|^{p} d x\right)^{1 / p}, \quad 1 \leqslant p<\infty, \\
\|f\|_{\infty} & =\underset{x \in[0,2 \pi]}{\operatorname{vrai} \sup }|f(x)| .
\end{aligned}
$$

При $1 \leqslant p<\infty$ будем изучать функции $f(x) \in L_{p}$, т.е. такие функции, у которых $\|f\|_{p}<\infty$. При $p=\infty$ будем изучать непрерывные функции $f(x) \in C$.

Изучаемые функции приближаются тригонометрическими полиномами

$$
P_{n}(x)=\sum_{k=-n}^{n} c_{k} e^{i k x}, \quad c_{k}=\bar{c}_{-k},
$$

т.е. тригонометрическими полиномами с вещественными коэффициентами.

Мы изучим оценки наилучших приближений

$$
E_{n}(f)_{p}=\inf _{P_{n}(x)}\left\|f(x)-P_{n}(x)\right\|_{p}
$$

через усредненные разности порядка $l$, т.е. через величины

$$
\Phi_{l}(f ; t)_{p}=\frac{1}{t} \int_{0}^{t}\left\|\Delta_{h}^{l} f(\cdot)\right\|_{p} d h .
$$


Аналогичные величины для $0<p<1$ были рассмотрены Руновским [1], [2]. В работе [3] доказано, что наилучшие приближения функции полиномами по системе Хаара оцениваются через усредненные разности $(0<p<1)$.

Рассмотрим также модули непрерывности порядка $l$, задаваемые равенством

$$
\omega_{l}(f ; t)_{p}=\sup _{|h| \leqslant t}\left\|\Delta_{h}^{l} f(\cdot)\right\|_{p}, \quad 1 \leqslant p \leqslant \infty .
$$

Очевидно, $\Phi_{l}(f ; t)_{p} \leqslant \omega_{l}(f ; t)_{p}$ при всех $l \geqslant 1$.

Величины $\Phi_{l}(f ; t)_{p}$ обладают следуюшими свойствами.

1) $\lim _{t \rightarrow 0} \Phi_{l}(f ; t)_{p}=0$. (Это свойство легко вытекает из правила Лопиталя.)

2) $\Phi_{l}(f ; t)_{p}$ непрерывна при $t>0$.

3) $\Phi_{l}\left(f_{1}+f_{2} ; t\right)_{p} \leqslant \Phi_{l}\left(f_{1} ; t\right)_{p}+\Phi_{l}\left(f_{2} ; t\right)_{p}$.

4) $\Phi_{l}(f ; t)_{p} \leqslant 2^{l}\|f\|_{p}$.

5) $\Phi_{l}(f ; n t)_{p} \leqslant n^{l} \Phi_{l}(f ; t)_{p}, n \in \mathbb{N}$.

Докажем это свойство.

$$
\Phi_{l}(f ; n t)_{p}=\frac{1}{n t} \int_{0}^{n t}\left\|\Delta_{h}^{l} f(\cdot)\right\|_{p} d h=\frac{1}{t} \int_{0}^{t}\left\|\Delta_{n \tau}^{l} f(\cdot)\right\|_{p} d \tau .
$$

Мы сделали замену $h=n \tau$. Далее, применяя равенство

$$
\Delta_{n \tau}^{l} f(x)=\sum_{i_{1}=0}^{n-1} \cdots \sum_{i_{l}=0}^{n-1} \Delta_{\tau}^{l} f\left(x+\left(i_{1}+\cdots+i_{l}\right) \tau\right)
$$

получаем свойство 5).

6) $\Phi_{l}(f ; t)_{p}$ почти возрастает, т.е. существует константа $c$, не зависящая от $t$, и такая, что для всех $0<t_{1}<t_{2}$ вьполняется неравенство

$$
\Phi_{l}\left(f ; t_{1}\right)_{p} \leqslant c \Phi_{l}\left(f ; t_{2}\right)_{p} .
$$

Это свойство следует из сформулированной ниже теоремы 1. Действительно,

$$
\Phi_{l}\left(f ; t_{1}\right)_{p} \leqslant \omega_{l}\left(f ; t_{1}\right)_{p} \leqslant \omega_{l}\left(f ; t_{2}\right)_{p} \leqslant c \Phi_{l}\left(f ; t_{2}\right)_{p} .
$$

Оценки наилучших приближений через усредненные разности рассматривались в уже упомянутых работах [1]-[3] (при $0<p<1)$. В работе Ульянова [4] в леммах 3 и $3^{\prime}$ такие оценки фактически доказаны для случая $1 \leqslant p \leqslant \infty, l=1$.

Следующая теорема показывает слабую эквивалентность величин $\Phi_{l}(f ; t)_{p}$ и $\omega_{l}(f ; t)_{p}$

ТЕОРема 1. Пусть $f(x)$ является $2 \pi$-периодической функиией из $L_{p}$, $1 \leqslant p<\infty$, либо из $C, p=\infty$. Тогда

$$
\Phi_{l}(f ; t)_{p} \leqslant \omega_{l}(f ; t)_{p} \ll \Phi_{l}(f ; t)_{p} .
$$

ЗАмечАниЕ 1. Первое неравенство очевидно, а второе выполняется с некоторой константой, не зависящей от $t$.

ЗАмечАниЕ 2. Доказательство теоремы 1 аналогично доказательству теоремы 3.1 из [2].

Из теоремы 1 и теоремы Джексона вытекает

Теорема 2. Для любой $2 \pi$-периодической функиии $f \in L_{p}, 1 \leqslant p<\infty$, либо $f \in C, p=\infty$, верно неравенство $E_{n}(f)_{p} \leqslant c \Phi_{l}(f ; 2 \pi / n)_{p}$, әде с не зависит om $n$. 


\section{$\S 2$.}

Перейдем к многомерному случаю.

В этом параграфе мы будем рассматривать функции от $d$ вешественных переменных $f(\mathbf{x})=f\left(x_{1}, \ldots, x_{d}\right)$, имеющие период $2 \pi$ по каждой переменной.

Пусть $\pi_{d}=[0,2 \pi]^{d}$, тогда

$$
\begin{array}{rlrl}
\|f\|_{p} & =\left(\frac{1}{(2 \pi)^{d}} \int_{\pi_{d}}|f(\mathbf{x})|^{p} d \mathbf{x}\right)^{1 / p}, & 1 \leqslant p<\infty, \\
\|f\|_{\infty} & =\operatorname{vrai~sup}_{\mathbf{x} \in \pi_{d}}|f(\mathbf{x})|, & & p=\infty .
\end{array}
$$

Будем, по-прежнему, считать, что $f(\mathbf{x}) \in L_{p}$ при $1 \leqslant p<\infty$ и $f(\mathbf{x}) \in C$ при $p=\infty$. Для $d$-мерного вектора $\mathbf{s}=\left(s_{1}, \ldots, s_{d}\right)$ с целыми неотрицательными компонентами через $\rho(\mathbf{s})$ будем обозначать двоичную пачку:

$$
\rho(\mathbf{s})=\left\{\mathbf{k}=\left(k_{1}, \ldots, k_{d}\right): k_{j} \text {-целые, } 2^{s_{j}-1} \leqslant\left|k_{j}\right|<2^{s_{j}}, j=1, \ldots, d\right\} .
$$

Пусть $\varkappa_{n}, n \in \mathbb{N},-$ такие множества, что

$$
\bigcup_{n=1}^{\infty} \varkappa_{n}=\mathbb{R}_{+}^{d}, \quad \varkappa_{n} \subset \varkappa_{n+1} .
$$

Предположим, что множества $\varkappa_{n}$ таковы, что для любого $\mathbf{s}=\left(s_{1}, \ldots, s_{\boldsymbol{d}}\right) \in \varkappa_{n}$ справедливо вложение

$$
\prod_{j=1}^{d}\left[0, s_{j}\right] \subset \varkappa_{n} .
$$

Обозначим $\theta_{n}=\varkappa_{n+1} \backslash \varkappa_{n}$. Считаем, что для любого вектора $\mathbf{s}=\left(s_{1}, \ldots, s_{d}\right)$, $s_{j} \in \mathbb{Z}_{+}$, такого, что $\mathbf{s} \in \varkappa_{n}$, и для любого индекса $1 \leqslant j_{0} \leqslant d$ сушествует $\tilde{s}_{j_{0}} \in \mathbb{Z}_{+}$, для которого $\left(s_{1}, \ldots, s_{j_{0}-1}, \tilde{s}_{j_{0}}, s_{j_{0}+1}, \ldots, s_{d}\right) \in \theta_{n}$. Кроме того, предполагаем сушествование числа $r>0$, не зависящего от $n$, и такого, что для любого $\mathbf{s} \in \theta_{n}$ и для любого индекса $1 \leqslant j_{0} \leqslant d$ вектор $\left(s_{1}, \ldots, s_{j_{0}-1}, s_{j_{0}}+r, s_{j_{0}+1}, \ldots, s_{d}\right)$ не принадлежит множеству $\theta_{n}$.

Исходя из множества $\varkappa_{n}$, определим множества

$$
Q_{n}=\bigcup_{\mathbf{s} \in \varkappa_{n}} \rho(\mathbf{s})
$$

Как легко видеть, множества $Q_{n}$ центрально симметричны.

Будем приближать функции $f(\mathbf{x})$ тригонометрическими полиномами с гармониками из $Q_{n}$, т.е. полиномами

$$
P_{n}(\mathbf{x})=\sum_{\mathbf{k} \in Q_{n}} c_{\mathbf{k}} e^{i(\mathbf{k}, \mathbf{x})}
$$


где $c_{-k_{1}, \ldots,-k_{d}}=\bar{c}_{k_{1}, \ldots, k_{d}}$, вектор $\mathbf{k}=\left(k_{1}, \ldots, k_{d}\right)$ имеет целочисленные координаты. Для таких полиномов будем писать

$$
P_{n}(\mathbf{x}) \in T\left(Q_{n}\right) .
$$

Определим наилучшие приближения функции $f(\mathbf{x})$

$$
E_{Q_{n}}(f)_{p}=\inf _{P_{n} \in T\left(Q_{n}\right)}\left\|f-P_{n}\right\|_{p}
$$

Смешанная разность порядка $l$ функции $f(\mathbf{x})$ определяется следуюшим образом $\left(\mathbf{h}=\left(h_{1}, \ldots, h_{d}\right)\right)$ :

$$
\Delta_{\mathbf{h}}^{l} f(\mathbf{x})=\Delta_{h_{1}}^{l} \cdots \Delta_{h_{d}}^{l} f(\mathbf{x}),
$$

т.е. это разность порядка $l$ с шагом $h_{j}$ по переменной $x_{j}$.

Изучим оценку наилучшего приближения $E_{Q_{n}}(f)_{p}$ через усредненные смешанные разности, т.е. величины

$$
\begin{aligned}
\Phi^{l}(f ; \mathbf{t})_{p} & =\Phi^{l}\left(f ;\left(t_{1}, \ldots, t_{d}\right)\right)_{p} \\
& =\frac{1}{t_{1} \cdots t_{d}} \int_{0}^{t_{1}} \cdots \int_{0}^{t_{d}}\left\|\Delta_{\mathbf{h}}^{l} f(\cdot)\right\|_{p} d h_{1} \ldots d h_{d} .
\end{aligned}
$$

Для этих величин справедливы свойства 1)-4), сформулированные в 1 . Свойство 5) запишется иначе:

$\left.5^{\prime}\right) \Phi^{l}\left(f ;\left(k_{1} t_{1}, \ldots, k_{d} t_{d}\right)\right)_{p} \leqslant\left(\prod_{j=1}^{d} k_{j}\right)^{l} \Phi^{l}(f ; \mathbf{t})_{p}$.

Свойство 6) примет следующий вид.

$6^{\prime}$ Существует константа $c$, не зависящая от $\mathbf{t}$ и такая, что если $\mathbf{t}^{(1)}=$ $\left(t_{1}^{(1)}, \ldots, t_{d}^{(1)}\right), \mathbf{t}^{(2)}=\left(t_{1}^{(2)}, \ldots, t_{d}^{(2)}\right), t_{j}^{(1)} \leqslant t_{j}^{(2)}, j=1, \ldots, d$, то $\Phi^{l}\left(f ; \mathbf{t}^{(1)}\right)_{p} \leqslant$ $c \Phi^{l}\left(f ; \mathbf{t}^{(2)}\right)_{p}$.

Это свойство легко получается из его одномерного аналога, из свойства 6$)$.

Отметим, что при фиксированных $t_{i}, i \neq j$, функция $\Phi^{l}\left(f ; t_{1}, \ldots, t_{j-1}, t_{j}\right.$, $\left.t_{j+1}, \ldots, t_{d}\right)_{p}$ как функция от $t_{j}$ обладает всеми свойствами одномерных усредненных разностей.

Теорема 1 легко обобщается на многомерный случай. Определим смешанный модуль непрерывности порядка $l$ следующим образом:

$$
\Omega^{l}(f ; \mathbf{t})_{p}=\sup _{\substack{\left|h_{j}\right| \leqslant t_{j} \\ j=1, \ldots, d}}\left\|\Delta_{\mathbf{h}}^{l} f(\mathbf{x})\right\|_{p},
$$

где разности $\Delta_{\mathbf{h}}^{l} f(\mathbf{x})$ определяются равенством (1).

Tеорема 3. Пусть $f(\mathbf{x}) \in L_{p}, 1 \leqslant p<\infty$, либо $f(\mathbf{x}) \in C, p=\infty$, и пусть $f(\mathbf{x})$ имеет период $2 \pi$ по каждой переменной. Тогда

$$
\Phi^{l}(f ; \mathbf{t})_{p} \leqslant \Omega^{l}(f ; \mathbf{t})_{p} \ll \Phi^{l}(f ; \mathbf{t})_{p},
$$

причем константа во втором неравенстве не зависит от $\mathbf{t}$.

Перейдем к оценке наилучших приближений. Всюду ниже $\mathbf{s}=\left(s_{1}, \ldots, s_{\boldsymbol{d}}\right)$ будет обозначать вектор с целыми неотрицательными компонентами. 
Пусть $e \subseteq\{1, \ldots, d\}$. Количество элементов множества $e$ удовлетворяет условию $|e|=\nu \leqslant d$. Для такого множества определим

$$
\Phi_{e}^{l}(f ; \mathbf{t})_{p}=\left(\prod_{j \in e} t_{j}\right)^{-1} \int_{0}^{t_{j_{1}}} \ldots \int_{0}^{t_{j_{\nu}}}\left\|\Delta_{\mathbf{h}}^{l}(e) f(\mathbf{x})\right\|_{p} d h_{j_{1}} \ldots d h_{j_{\nu}},
$$

где

$$
\Delta_{\mathbf{h}}^{l}(e) f(\mathbf{x})=\left(\prod_{j \in e} \Delta_{h_{j}}^{l}\right) f(\mathbf{x})
$$

- разность порядка $l$ лишь по переменным $x_{j}, j \in e$, с шагом $h_{j}$. Положим

$$
\theta_{n}^{(e)}=\left\{\mathbf{s} \in \theta_{n}: s_{j}=0, j \notin e\right\} .
$$

Если $e=\{1, \ldots, d\}$, то $e$ указьвать не будем.

Tеорема 4. Пусть функиия $f(\mathbf{x})=f\left(x_{1}, \ldots, x_{d}\right)$ является $2 \pi$-периодической по каждой переменной. Пусть, далее, $f(\mathbf{x}) \in L_{p}, 1 \leqslant p<\infty$, либо $f(\mathbf{x}) \in C, p=\infty$. Тогда

$$
\begin{aligned}
& E_{Q_{n}}(f)_{p} \ll \sum_{1 \leqslant|e| \leqslant d}\left(\sum_{\mathbf{s} \in \theta_{n}^{(e)}}\left(\Phi_{e}^{l}\left(f ; 2^{-\mathbf{s}}\right)_{p}\right)^{p_{0}}\right)^{1 / p_{0}}, 1 \leqslant p<\infty, \\
& E_{Q_{n}}(f)_{\infty} \ll \sum_{1 \leqslant|e| \leqslant d} \sum_{\mathbf{s} \in \theta_{n}^{(e)}} \Phi_{e}^{l}\left(f ; 2^{-\mathbf{s}}\right)_{\infty},
\end{aligned}
$$

где $2^{-\mathbf{s}}=\left(2^{-s_{1}}, \ldots, 2^{-s_{d}}\right), p_{0}=\min \{p ; 2\}$. Указаннье неравенства выполняются с константами, не зависящими от $n$.

ДоказАтельство. СЛучай А) $1<p<\infty$. Доказательство аналогично доказательству многомерной теоремы Джексона из работы [5].

Сначала рассмотрим функции $f(\mathbf{x}) \in L_{p}^{0}$, т.е. такие $f(\mathbf{x})$, что

$$
\int_{0}^{2 \pi} f(\mathbf{x}) d x_{j}=0, \quad j=1, \ldots, d .
$$

Пусть $1<p \leqslant 2$. Тогда $p_{0}=p$. Как известно, наилучшие по порядку приближения в рассматриваемом случае дают суммы Фурье. Поэтому для $f \in L_{p}$ имеем

$$
E_{Q_{n}}(f)_{p} \ll\left\|\sum_{\mathbf{m} \notin \varkappa_{n}} \delta_{\mathbf{m}}(f ; \mathbf{x})\right\|_{p}
$$

где

$$
\delta_{\mathbf{m}}(f ; \mathbf{x})=\sum_{\mathbf{k} \in \rho(\mathbf{m})} \hat{f}(\mathbf{k}) e^{i(\mathbf{k}, \mathbf{x})},
$$

$\hat{f}(\mathbf{k})$ - коэффициенты Фурье функции $f(\mathbf{x}), \mathbf{m}=\left(m_{1}, \ldots, m_{d}\right), m_{j} \in \mathbb{Z}_{+}$. В силу условий, наложенных на множества $\varkappa_{n}$, найдется число $\alpha_{1}=\alpha_{1}(l, d)$ такое, что 
для любого $\mathbf{m} \notin \varkappa_{n+\alpha_{1}}, m_{j}>l+3$, сушествует $\mathbf{s}=\left(s_{1}, \ldots, s_{d}\right) \in \theta_{n}$ такое, что $m_{j} \geqslant s_{j}+l+3, j=1, \ldots, d$. Обозначим это $\mathbf{s}$ через $\mathbf{s}(\mathbf{m})$.

Если для некоторого вектора $\mathbf{m}=\left(m_{1}, \ldots, m_{d}\right)$ с целыми неотрицательными компонентами, $\mathbf{m} \in \varkappa_{n+\alpha_{1}}$, найдется индекс $1 \leqslant j_{0} \leqslant d$ такой, что $m_{j_{0}} \leqslant l+3$, то можно утверждать, что сушествует $1 \leqslant j_{1} \leqslant d$, для которого $m_{j_{1}}>l+3$. Более точно, множество $\{1, \ldots, d\}$ можно разбить на два непересекаюшихся множества $e_{1}$ и $e_{2}$ такие, что $m_{j} \leqslant l+3$ для $j \in e_{1}$ и $m_{j}>l+3$ для $j \in e_{2}$, причем множество $e_{2}$ непусто.

Можно указать такое $\alpha_{2}=\alpha_{2}(l, d)$, что при $\mathbf{m} \notin \varkappa_{n+\alpha_{2}}$, для которого $m_{j} \leqslant$ $l+3, j \in e_{1}$, и $m_{j}>l+3, j \in e_{2}$, будет существовать $\mathbf{s} \in \theta_{n}^{\left(e_{2}\right)}$ такое, что $m_{j} \geqslant s_{j}+l+3, j \in e_{2}$. Это $\mathbf{s}$ мы также будем обозначать $\mathbf{s}(\mathbf{m})$.

Возьмем $\alpha=\max \left\{\alpha_{1} ; \alpha_{2}\right\}$. Обозначим

$$
\begin{aligned}
& A_{n l}(\alpha)=\left\{\mathbf{m}: m_{j}>0 \text { - целые, } m_{j}>l+3, j=1, \ldots, d, \mathbf{m} \notin \varkappa_{n+\alpha}\right\} . \\
& B_{n l}(\alpha)=\left\{\mathbf{m}: m_{j} \geqslant 0 \text { - целые, } m_{j} \leqslant l+3, j \in e_{1} \neq \varnothing,\right. \\
&\left.m_{j}>l+3, j \in e_{2}, \mathbf{m} \notin \varkappa_{n+\alpha}\right\} .
\end{aligned}
$$

Далее, очевидно,

$$
\begin{aligned}
E_{Q_{n}}(f)_{p} \ll & \sum_{\mathbf{m} \in \varkappa_{n+\alpha} \backslash \varkappa_{n}} \delta_{\mathbf{m}}(f ; \mathbf{x}) \|_{p} \\
& +\left\|\sum_{\mathbf{m} \in A_{n l}(\alpha)} \delta_{\mathbf{m}}(f ; \mathbf{x})\right\|_{p}+\left\|\sum_{\mathbf{m} \in B_{n l}(\alpha)} \delta_{\mathbf{m}}(f ; \mathbf{x})\right\|_{p} .
\end{aligned}
$$

Оценим второе слагаемое. Как показано в [5], числа $\left(\lambda_{k_{j}}^{(l)}\right)^{-1}$, обратные числам

$$
\lambda_{k_{j}}^{(l)}=2^{s_{j}(\mathbf{m})} \int_{0}^{2^{-s_{j}(\mathbf{m})}}\left(e^{i k_{j} t_{j}}-1\right)^{l} d t_{j}, \quad\left[2^{m_{j}-1}\right] \leqslant\left|k_{j}\right|<2^{m_{j}}
$$

являются множителями Марцинкевича. По теореме о множителях Марцинкевича (см. [6, теорема Б]) второе слагаемое оценивается следующим образом:

$$
\left\|\sum_{\mathbf{m} \in A_{n l}(\alpha)} \delta_{\mathbf{m}}(f ; \mathbf{x})\right\|_{p} \ll \sum_{\mathbf{m} \in A_{n l}(\alpha)} \sum_{\mathbf{k} \in \rho(\mathbf{m})} \hat{f}(\mathbf{k}) \prod_{j=1}^{d} \lambda_{k_{j}}^{(l)} e^{i(\mathbf{k}, \mathbf{x})} \|_{p}=I .
$$

Обозначим

$$
\Phi_{\mathbf{s}}(\mathbf{x})=\int_{0}^{2^{-s_{1}}} \cdots \int_{0}^{2^{-s_{d}}} \Delta_{\mathbf{t}}^{l} f(\mathbf{x}) d \mathbf{t}
$$

Считаем, далее, $\|\mathbf{s}\|_{1}=s_{1}+\cdots+s_{d}$. Используя теорему Литтлвуда-Пэли, получаем

$$
\begin{aligned}
I & \ll\left\|\left(\sum_{\mathbf{m} \in A_{n l}(\alpha)}\left|2^{\|\mathbf{s}(\mathbf{m})\|_{1}} \delta_{\mathbf{m}}\left(\Phi_{\mathbf{s}(\mathbf{m})} ; \mathbf{x}\right)\right|^{2}\right)^{1 / 2}\right\|_{p} \\
& \ll\left(\sum_{\mathbf{s} \in \theta_{n}}\left\|\left(\sum_{\mathbf{m}}\left|\delta_{\mathbf{m}}\left(2^{\|\mathbf{s}\|_{1}} \Phi_{\mathbf{s}} ; \mathbf{x}\right)\right|^{2}\right)^{1 / 2}\right\|_{p}^{p}\right)^{1 / p} .
\end{aligned}
$$


Здесь внутренняя сумма берется по всем векторам $\mathbf{m}=\left(m_{1}, \ldots, m_{d}\right)$ с целыми неотрицательньми компонентами. Применяя к этому выражению теорему Литтлвуда-Пэли, а затем обобщенное неравенство Минковского, мы получаем

$$
\left\|\sum_{\mathbf{m} \in A_{n l}(\alpha)} \delta_{\mathbf{m}}(f ; \mathbf{x})\right\|_{p} \ll\left(\sum_{\mathbf{s} \in \theta_{n}}\left(\Phi^{l}\left(f ; 2^{-\mathbf{s}}\right)_{p}\right)^{p}\right)^{1 / p} .
$$

Оценим последнее слагаемое в неравенстве (3). Воспользуемся, как и при оценке второго слагаемого, множителями Марцинкевича $\left(\lambda_{k_{j}}^{(l)}\right)^{-1}$, однако будем применять мультипликаторные преобразования лишь по переменным $x_{j}, j \in e_{2}$ (т.е. для таких $j$, что $m_{j} \geqslant(\mathbf{s}(\mathbf{m}))_{j}+l+3$ при $\left.\mathbf{m} \in B_{n l(\alpha)}\right)$.

Введем следуюшие обозначения: для $\mathbf{s} \in \theta_{n}^{\left(e_{2}\right)}$ положим

$$
\sigma(\mathbf{s})=\prod_{j \in e_{2}}\left[0,2^{-s_{j}}\right]
$$

$\mathbf{t}\left(e_{i}\right)$ обозначает $d$-мерньй вектор $\left(t_{1}, \ldots, t_{d}\right)$, у которого $t_{j}>0$ при $j \in e_{i}$ и $t_{j}=0$ при $j \neq e_{i}(i=1,2)$. Положим

$$
d \mathbf{t}\left(e_{i}\right)=\prod_{j \in e_{i}} d t_{j}
$$

По теореме Марцинкевича о мультипликаторах имеем

$$
\left\|\sum_{\mathbf{m} \in B_{n l}(\alpha)} \delta_{\mathbf{m}}(f ; \mathbf{x})\right\|_{p} \ll \sum_{\mathbf{m} \in B_{n l}(\alpha)} 2^{\|\mathbf{s}(\mathbf{m})\|_{1}} \delta_{\mathbf{m}}\left(\Phi_{\mathbf{s}(\mathbf{m})} ; \mathbf{x}\right) \|_{p} .
$$

Здесь $\mathbf{s}(\mathbf{m}) \in \theta_{n}^{\left(e_{2}\right)}$,

$$
\Phi_{\mathbf{s}(\mathbf{m})}(\mathbf{x})=\int_{\sigma(\mathbf{s}(\mathbf{m}))} \Delta_{\mathbf{t}}^{l}\left(e_{2}\right) f(\mathbf{x}) d \mathbf{t}\left(e_{2}\right) .
$$

Так как $f \in L_{p}^{0}$, то верно равенство

$$
f(\mathbf{x})=\frac{(-1)^{l}}{2 \pi} \int_{0}^{2 \pi} \Delta_{t_{j}}^{l} f(\mathbf{x}) d t_{j}, \quad j=1, \ldots, d
$$

где разность $\Delta_{t_{j}}^{l}$ берется по соответствуюшей переменной $x_{j}$. Положим, далее, $\left|e_{2}\right|=\nu$. В силу вьшесказанного

$$
\Phi_{\mathbf{s}(\mathbf{m})}(\mathbf{x})=\frac{(-1)^{l(d-\nu)}}{(2 \pi)^{d-\nu}} \int_{\pi_{d-\nu}}\left(\int_{\sigma(\mathbf{s}(\mathbf{m}))} \Delta_{\mathbf{t}}^{l} f(\mathbf{x}) d \mathbf{t}\left(e_{2}\right)\right) d \mathbf{t}\left(e_{1}\right) .
$$

Пусть $\mathbf{s} \in \theta_{n}^{\left(e_{2}\right)}$. Возьмем вектор $\mathbf{s}^{\prime}=\left(s_{1}^{\prime}, \ldots, s_{d}^{\prime}\right)$ такой, что $s_{j}^{\prime}=s_{j}, j \in e_{2}$, и $s_{j}^{\prime}=1, j \in e_{1}$. Множество всех векторов $\mathbf{s}^{\prime}$ таких, что $\mathbf{s} \in \theta_{n}^{\left(e_{2}\right)}$, обозначим $\gamma_{n}\left(e_{2}\right)$. 
Аналогично доказательству оценки второго слагаемого после применения теоремы Литтлвуда-Пэли и обобщенного неравенства Минковского мы придем к неравенству

$$
\begin{aligned}
\left\|\sum_{\mathbf{m} \in B_{n l}(\alpha)} \delta_{\mathbf{m}}(f ; \mathbf{x})\right\|_{p} & \ll\left(\sum_{\mathbf{s} \in \gamma_{n}\left(e_{2}\right)}\left(\Phi^{l}\left(f ; 2^{-\mathbf{s}}\right)_{p}\right)^{p}\right)^{1 / p} \\
& \ll\left(\sum_{\mathbf{s} \in \theta_{n}}\left(\Phi^{l}\left(f ; 2^{-\mathbf{s}}\right)_{p}\right)^{p}\right)^{1 / p} .
\end{aligned}
$$

Последнее неравенство легко следует из свойств $\left.5^{\prime}\right)$ и $\left.6^{\prime}\right)$ функции $\Phi^{l}(f ; \mathbf{t})_{p}$.

Из условий, наложенных на множества $\theta_{n}$, и из свойств функции $\Phi^{l}(f ; \mathbf{t})_{p}$ вытекает следуюшая оценка первого слагаемого:

$$
\left\|\sum_{\mathbf{m} \in \varkappa_{n+\alpha} \backslash \varkappa_{n}} \delta_{\mathbf{m}}(f ; \mathbf{x})\right\|_{p} \ll\left(\sum_{\mathbf{s} \in \theta_{n}}\left(\Phi^{l}\left(f ; 2^{-\mathbf{s}}\right)_{p}\right)^{p}\right)^{1 / p} .
$$

Из доказанных оценок следует, что

$$
E_{Q_{n}}(f)_{p} \ll\left(\sum_{\mathbf{s} \in \theta_{n}}\left(\Phi^{l}\left(f ; 2^{-\mathbf{s}}\right)_{p}\right)^{p}\right)^{1 / p} .
$$

Случай $1<p \leqslant 2$ рассмотрен.

Продолжим рассмотрение случая А). По прежнему, считаем $f(\mathbf{x}) \in L_{p}^{0}$.

Пусть теперь $2<p<\infty$. В этом случае $p_{0}=2$, неравенство (3) остается верным. Покажем, как оценивается второе слагаемое в правой части (3). Справедливо следуюшее:

$$
\begin{aligned}
\left\|\sum_{\mathbf{m} \in A_{n l}(\alpha)} \delta_{\mathbf{m}}(f ; \mathbf{x})\right\|_{p} & \ll\left\|\left(\sum_{\mathbf{m} \in A_{n l}(\alpha)}\left|2^{\|\mathbf{s}(\mathbf{m})\|_{1}} \delta_{\mathbf{m}}\left(\Phi_{\mathbf{s}(\mathbf{m})} ; \mathbf{x}\right)\right|^{2}\right)^{1 / 2}\right\|_{p} \\
& \ll\left(\left\|\sum_{s \in \theta_{n}} \sum_{\mathbf{m}}\left|2^{2\|\mathbf{s}\|_{1}} \delta_{\mathbf{m}}\left(\Phi_{\mathbf{s}} ; \mathbf{x}\right)\right|^{2}\right\|_{p / 2}\right)^{1 / 2} .
\end{aligned}
$$

(Первое неравенство получается аналогично случаю $1<p \leqslant 2$, при получении второго неравенства учитываем, что $p>2$.) Затем, применяя неравенство Минковского, теорему Литтлвуда-Пэли и, наконец, обобщенное неравенство Минковского, приходим к оценке

$$
\left\|\sum_{\mathbf{m} \in A_{n l}(\alpha)} \delta_{\mathbf{m}}(f ; \mathbf{x})\right\|_{p} \ll\left(\sum_{\mathbf{s} \in \theta_{n}}\left(\Phi^{l}\left(f ; 2^{-\mathbf{s}}\right)_{p}\right)^{2}\right)^{1 / 2} .
$$

C учетом того, что $p>2$, оценка последнего слагаемого из правой части (3) примет вид:

$$
\begin{aligned}
\left\|\sum_{\mathbf{m} \in B_{n l}(\alpha)} \delta_{\mathbf{m}}(f ; \mathbf{x})\right\|_{p} & \ll\left(\sum_{\mathbf{s} \in \gamma_{n}\left(e_{2}\right)}\left(\Phi^{l}\left(f ; 2^{-\mathbf{s}}\right)_{p}\right)^{2}\right)^{1 / 2} \\
& \ll\left(\sum_{\mathbf{s} \in \theta_{n}}\left(\Phi^{l}\left(f ; 2^{-\mathbf{s}}\right)_{p}\right)^{2}\right)^{1 / 2} .
\end{aligned}
$$


Наконец, первое слагаемое оценивается с помощью свойств $\Phi^{l}(f ; \mathbf{t})_{p}$ и условий, которьм удовлетворяют множества $\theta_{n}$.

С учетом всего сказанного имеем

$$
E_{Q_{n}}(f)_{p} \ll\left(\sum_{\mathbf{s} \in \theta_{n}}\left(\Phi^{l}\left(f ; 2^{-\mathbf{s}}\right)_{p}\right)^{2}\right)^{1 / 2}
$$

Случай $2<p<\infty, f \in L_{p}^{0}$ рассмотрен. Переход от $L_{p}^{0}$ к $L_{p}$ осушествляется аналогично тому, как это было сделано в работе [5]. Случай А) рассмотрен полностью.

Случай Б) $p=1, \infty$. В этих случаях доказательство аналогично доказательству многомерной теоремы Джексона из [7].

Для вектора с натуральными компонентами $\mathbf{m}=\left(m_{1}, \ldots, m_{d}\right)$ обозначим через $U(\mathbf{m})$ множество функций

$$
u(\mathbf{x})=\sum_{j=1}^{d} \sum_{\nu_{j}=-m_{j}}^{m_{j}} e^{i \nu_{j} x_{j}} C\left(x_{1}, \ldots, x_{j-1}, x_{j+1}, \ldots, x_{d}\right),
$$

где $C\left(x_{1}, \ldots, x_{j-1}, x_{j+1}, \ldots, x_{d}\right)$ - непрерывная и $2 \pi$-периодическая по каждой переменной функция. Для $f(\mathbf{x}) \in L_{p}^{0}$ докажем следующую лемму.

Лемма 1. Для всякой функиии $f(\mathbf{x}) \in L_{p}^{0}, 1 \leqslant p \leqslant \infty$, имеющей период $2 \pi$ по каждой переменной, существует функиия $u(\mathbf{x}) \in U(\mathbf{m})$, для которой

$$
\|f-u\|_{p} \ll \Phi^{l}\left(f ; \frac{1}{\mathbf{m}}\right)_{p}
$$

əде $1 / \mathbf{m}=\left(1 / m_{1}, \ldots, 1 / m_{d}\right)$.

ДОКАЗАТЕЛЬСТВо ЛЕмМЫ 1. Положим

$$
u_{1}(\mathbf{x})=\frac{1}{\pi} \int_{-\pi}^{\pi}\left(\int_{0}^{\left|t_{1}\right|}\left((-1)^{l} f(\mathbf{x})-\Delta_{h_{1}}^{l} f(\mathbf{x})\right) d h_{1}\right) J_{m_{1}}^{l+1}\left(t_{1}\right) d t_{1}
$$

Как легко видеть, $u_{1}(\mathbf{x}) \in U(\mathbf{m})$ (здесь $J_{m_{1}}^{l+1}\left(t_{1}\right)-$ (одномерное) ядро Джексона порядка $\left.m_{1}\right)$. Далее, пусть

$$
f_{1}(\mathbf{x})=f(\mathbf{x})-\frac{u_{1}(\mathbf{x})}{\beta_{1}\left(m_{1} ; l\right)} \cdot(-1)^{l}
$$

где

$$
\beta_{1}\left(m_{1} ; l\right)=\frac{1}{\pi} \int_{-\pi}^{\pi}\left|t_{1}\right| J_{m_{1}}^{l+1}\left(t_{1}\right) d t_{1}
$$

Из свойств ядра Джексона следует, что $\beta_{1}\left(m_{1} ; l\right) \asymp 1 / m_{1}$. 
Справедливо следующее:

$$
\begin{aligned}
&\left\|\left(\prod_{j=2}^{d} \frac{1}{t_{j}}\right) \int_{0}^{t_{2}} \cdots \int_{0}^{t_{d}} \Delta_{h_{2}}^{l} \cdots \Delta_{h_{d}}^{l} f_{1}(\mathbf{x}) d h_{2} \cdots d h_{d}\right\|_{p} \\
&=\|\left(\prod_{j=2}^{d} \frac{1}{t_{j}}\right) \int_{0}^{t_{2}} \cdots \int_{0}^{t_{d}}\left(\frac{1}{\pi} \int_{-\pi}^{\pi}\left(\frac{1}{\beta_{1}\left(m_{1} ; l\right)} \int_{0}^{\left|t_{1}\right|} \Delta_{\mathbf{h}}^{l} f(\mathbf{x}) d h_{1}\right)\right. \\
&\left.\times J_{m_{1}}^{l+1}\left(t_{1}\right) d t_{1}\right) d h_{2} \cdots d h_{d} \|_{p} \\
& \ll \int_{-\pi}^{\pi} m_{1}\left|t_{1}\right| \Phi^{l}\left(f ;\left(\left|t_{1}\right|, t_{2}, \ldots, t_{d}\right)\right)_{p} J_{m_{1}}^{l+1}\left(t_{1}\right) d t_{1} \\
&= \int_{-1 / m_{1}}^{1 / m_{1}} m_{1}\left|t_{1}\right| \Phi^{l}\left(f ;\left(\left|t_{1}\right|, t_{2}, \ldots, t_{d}\right)\right)_{p} J_{m_{1}}^{l+1}\left(t_{1}\right) d t_{1} \\
&+\int_{1 / m_{1} \leqslant\left|t_{1}\right| \leqslant \pi} m_{1}\left|t_{1}\right| \Phi^{l}\left(f ;\left(\left|t_{1}\right|, t_{2}, \ldots, t_{d}\right)\right)_{p} J_{m_{1}}^{l+1}\left(t_{1}\right) d t_{1} \\
& \ll m_{1} \cdot \frac{1}{m_{1}} \cdot \Phi^{l}\left(f ;\left(\frac{1}{m_{1}}, t_{2}, \ldots, t_{d}\right)\right)_{p} \int_{-\pi}^{\pi} J_{m_{1}}^{l+1}\left(t_{1}\right) d t_{1} \\
&+m_{1}^{l+1} \Phi^{l}\left(f ;\left(\frac{1}{m_{1}}, t_{2}, \ldots, t_{d}\right)\right)_{p} \int_{-\pi}^{\pi}\left|t_{1}\right|^{l+1} J_{m_{1}}^{l+1}\left(t_{1}\right) d t_{1} \\
& \ll \Phi^{l}\left(f ;\left(\frac{1}{m_{1}}, t_{2}, \ldots, t_{d}\right)\right)_{p} \cdot
\end{aligned}
$$

Здесь мы использовали оценку $\beta_{1}\left(m_{1} ; l\right)$, обобшенное неравенство Минковского и свойства функции $\Phi^{l}(f ; \mathbf{t})_{p}$, а также свойства ядра Джексона.

Далее, положим

$$
u_{2}(\mathbf{x})=\frac{1}{\pi} \int_{-\pi}^{\pi}\left(\int_{0}^{\left|t_{2}\right|}\left((-1)^{l} f_{1}(\mathbf{x})-\Delta_{h_{2}}^{l} f_{1}(\mathbf{x})\right) d h_{2}\right) J_{m_{2}}^{l+1}\left(t_{2}\right) d t_{2}
$$

Пусть

$$
f_{2}(\mathbf{x})=f_{1}(\mathbf{x})-\frac{u_{2}(\mathbf{x})}{\beta_{2}\left(m_{2} ; l\right)} \cdot(-1)^{l}
$$

где

$$
\beta_{2}\left(m_{2} ; l\right)=\frac{1}{\pi} \int_{-\pi}^{\pi}\left|t_{2}\right| J_{m_{2}}^{l+1}\left(t_{2}\right) d t_{2} .
$$

Аналогично предыдущему получим неравенство

$$
\begin{aligned}
\|\left(\prod_{j=3}^{d} \frac{1}{t_{j}}\right) \int_{0}^{t_{3}} \cdots \int_{0}^{t_{d}} \Delta_{h_{3}}^{l} \cdots \Delta_{h_{d}}^{l} f_{2}(\mathbf{x}) & d h_{3} \cdots d h_{d} \|_{p} \\
& \ll \Phi^{l}\left(f ;\left(\frac{1}{m_{1}}, \frac{1}{m_{2}}, t_{3}, \ldots, t_{d}\right)\right)_{p} .
\end{aligned}
$$


Продолжая дальше этот процесс, мы придем к функции

$$
f_{d}(\mathbf{x})=f_{d-1}(\mathbf{x})-\frac{u_{d}(\mathbf{x})}{\beta_{d}\left(m_{d} ; l\right)} \cdot(-1)^{l},
$$

причем

$$
\left\|f_{d}(\mathbf{x})\right\|_{p} \ll \Phi^{l}\left(f ;\left(\frac{1}{m_{1}}, \ldots, \frac{1}{m_{d}}\right)\right)_{p} .
$$

Так как

$$
f(\mathbf{x})=f_{d}(\mathbf{x})+\sum_{j=1}^{d} \frac{u_{j}(\mathbf{x})}{\beta_{j}\left(m_{j}, l\right)} \cdot(-1)^{l},
$$

где

$$
(-1)^{l} \sum_{j=1}^{d} \frac{u_{j}(\mathbf{x})}{\beta_{j}\left(m_{j} ; l\right)} \in U(\mathbf{m})
$$

мы приходим к утверждению леммы 1.

Продолжим доказательство теоремы 4. Пусть $V_{N}(t)$ - (одномерное) ядро Валле-Пуссена порядка $2 N-1$; пусть, далее, $A_{0}=V_{0}=1, A_{m}=V_{2^{m-1}}-V_{2^{m-2}}, m-$ натуральное, $V_{2^{-1}}=V_{0}$.

Для вектора $\mathbf{s}=\left(s_{1}, \ldots, s_{d}\right)$ с цельми неотрицательными компонентами положим

$$
A_{\mathrm{s}}=\prod_{j=1}^{d} A_{s_{j}}
$$

Введем обозначения:

$$
V_{Q_{n}}^{d}=\sum_{\mathbf{s} \in \varkappa_{n}} A_{\mathbf{s}}
$$

$V_{Q_{n}}^{d}(f ; \mathbf{x})$ - свертка функции $f(\mathbf{x})=f\left(x_{1}, \ldots, x_{d}\right)$ с ядром $V_{Q_{n}}^{d}(\mathbf{x})$.

Оценим норму $(p=1, \infty)$

$$
\left\|f(\mathbf{x})-V_{Q_{n}}^{d}(f ; \mathbf{x})\right\|_{p}
$$

Считаем, что $f(\mathbf{x}) \in L_{p}^{0}$. Будем доказывать утверждение теоремы индукцией по размерности $d$. Для $d=1$ получим

$$
\sum_{s=0}^{n} A_{s}=V_{2^{n-1}}
$$

Как известно, суммы Валле-Пуссена дают наилучшие по порядку приближения. С учетом этого и в силу теоремы 2 верно неравенство

$$
\left\|f(x)-V_{2^{n-1}}(f ; x)\right\|_{p} \ll \Phi_{l}\left(f ; 2^{-n}\right)_{p} .
$$


Предположим, что требуемая оценка для (4) верна для размерностей $1, \ldots, d-1$. Докажем ее для размерности $d$. Обозначая через $I_{j}$ тождественный оператор, действуюший по переменной $x_{j}$, мы можем записать

$$
\begin{aligned}
I-V_{Q_{n}}^{d} & =\prod_{j=1}^{d} I_{j}-\sum_{\mathbf{s} \in \varkappa_{n}} A_{\mathbf{s}} \\
& =\left(\prod_{j=1}^{d-1} I_{j}-V_{Q_{n}}^{d-1}\right) I_{d}+\sum_{\mathbf{s}^{d}:\left(s_{1}, \ldots, s_{d-1}, 0\right) \in \varkappa_{n}} A_{\mathbf{s}^{d}}\left(I_{d}-V_{2^{s_{n}\left(\mathbf{s}^{d}\right)-1}}\right) .
\end{aligned}
$$

Здесь $\mathbf{s}^{d}=\left(s_{1}, \ldots, s_{d-1}\right)$,

$$
V_{Q_{n}}^{d-1}=\sum_{\mathbf{s}^{d}:\left(s_{1}, \ldots, s_{d-1}, 0\right) \in \varkappa_{n}} A_{\mathbf{s}^{d}}, \quad s_{n}\left(\mathbf{s}^{d}\right)=\max \left\{s_{d} \in \mathbb{Z}_{+}: \mathbf{s} \in \varkappa_{n}\right\}
$$

Из леммы 1 можно вывести следуюшую оценку для каждого слагаемого во второй сумме в правой части (5)

$$
\left\|A_{\mathbf{s}^{d}}\left(I_{d}-V_{2^{s_{n}\left(\mathbf{s}^{d}\right)-1}}\right) f\right\|_{p} \ll \Phi^{l}\left(f ; 2^{-\left(\mathbf{s}^{d}, s_{n}\left(\mathbf{s}^{d}\right)\right)}\right)_{p} .
$$

Пусть $f(\mathbf{x}) \in L_{p}^{0}$. Следовательно,

$$
\Delta_{h_{1}}^{l} \cdots \Delta_{h_{d-1}}^{l} f(\mathbf{x})=(-1)^{l} \frac{1}{2 \pi} \int_{0}^{2 \pi} \Delta_{\mathbf{h}}^{l} f(\mathbf{x}) d h_{d} .
$$

Из этого равенства, в свою очередь, следует, что

$$
\begin{aligned}
& \left\|\left(\prod_{j=1}^{d-1} \frac{1}{t_{j}}\right) \int_{0}^{t_{1}} \cdots \int_{0}^{t_{d-1}} \Delta_{h_{1}}^{l} \cdots \Delta_{h_{d-1}}^{l} f(\mathbf{x}) d h_{1} \cdots d h_{d-1}\right\|_{p} \\
& \quad \leqslant \Phi^{l}\left(f ;\left(t_{1}, \ldots, t_{d-1}, 2 \pi\right)\right)_{p} .
\end{aligned}
$$

В силу предположения индукции и свойств функции $\Phi^{l}(f ; \mathbf{t})_{p}$ из $(7)$ следует оценка

$$
\left\|\left(\prod_{j=1}^{d-1} I_{j}-V_{Q_{n}}^{d-1}\right) I_{d} f\right\|_{p} \ll \sum_{\mathbf{s} \in \theta_{n}, s_{d}=0} \Phi^{l}\left(f ; 2^{-\mathbf{s}}\right)_{p} .
$$

Из неравенств (6) и (8) получаем

$$
\left\|f(\mathbf{x})-V_{Q_{n}}^{d}(f ; \mathbf{x})\right\|_{p} \ll \sum_{\mathbf{s} \in \theta_{n}} \Phi^{l}\left(f ; 2^{-\mathbf{s}}\right)_{p} .
$$

Переход от $L_{p}^{0}$ к $L_{p}$ осуществляется так же, как в [7]. Теорема 4 полностью доказана. 


\section{$\S 3$.}

В этом параграфе будет сформулирована многомерная теорема Джексона.

По-прежнему, $E_{Q_{n}}(f)_{p}$ означает наилучшее приближение функции $f(\mathbf{x})$ из $L_{p}$ тригонометрическими полиномами с гармониками из множеств $Q_{n}$.

В случае, когда $Q_{n}$ - гиперболические кресты, многомерная теорема Джексона для $p=\infty$ была доказана в [7], а для $1<p<\infty$ она была доказана в [5]. Случай $p=1$ не был доказан.

Мы же рассмотрим вместо гиперболических крестов множества $Q_{n}$, порожденные поверхностями уровня функции $\Omega^{l}(f ; \mathbf{t})_{p}$, где

$$
\Omega^{l}(f ; \mathbf{t})_{p}=\sup _{\left|h_{j}\right| \leqslant t_{j}}\left\|\Delta_{\mathbf{h}}^{l} f(\mathbf{x})\right\|_{p}
$$

- модуль непрерывности порядка $l$ функции $f(\mathbf{x})$ (см. (1) и (2)).

Для $\mathbf{s}=\left(s_{1}, \ldots, s_{\boldsymbol{d}}\right)$, по-прежнему, считаем $2^{-\mathbf{s}}=\left(2^{-s_{1}}, \ldots, 2^{-s_{d}}\right)$. Для натурального числа $N$ положим

$$
\begin{gathered}
\varkappa(N)=\left\{\mathbf{s}=\left(s_{1}, \ldots, s_{d}\right): s_{j} \geqslant 0 \text { - целые, } \Omega^{l}\left(f ; 2^{-\mathbf{s}}\right)_{p} \geqslant \frac{1}{N}\right\}, \\
Q(N)=\bigcup_{\mathbf{s} \in \varkappa(N)} \rho(\mathbf{s}), \quad \theta(N)=\varkappa\left(2^{l} N\right) \backslash \varkappa(N) .
\end{gathered}
$$

Из свойств функции $\Omega^{l}(f ; \mathbf{t})_{p}$

1) $\Omega^{l}\left(f ;\left(t_{1}^{(1)}, \ldots, t_{d}^{(1)}\right)\right)_{p} \leqslant \Omega^{l}\left(f ;\left(t_{1}^{(2)}, \ldots, t_{d}^{(2)}\right)\right)_{p}, \quad t_{j}^{(1)} \leqslant t_{j}^{(2)}$,

2) $\Omega^{l}\left(f ;\left(k_{1} t_{1}, \ldots, k_{d} t_{d}\right)\right)_{p} \leqslant \prod_{j=1}^{d} k_{j}^{l} \Omega^{l}(f ; \mathbf{t})_{p}, \quad k_{j} \in \mathbb{N}$

легко следует, что для любого $\mathbf{s}=\left(s_{1}, \ldots, s_{d}\right) \in \varkappa(N)$, для любого $1 \leqslant j_{0} \leqslant d$ сушествует $\tilde{s}_{j_{0}}$ такое, что

$$
\left(s_{1}, \ldots, s_{j_{0}-1}, \tilde{s}_{j_{0}}, s_{j_{0}+1}, \ldots, s_{d}\right) \in \theta(N) .
$$

Гиперболические кресты - это множества, порожденные поверхностями уровня функции

$$
\Omega(\mathbf{t})=\prod_{j=1}^{d} t_{j}^{r_{j}} .
$$

Для такого $\Omega(\mathbf{t})$ класс $H_{p}^{\Omega}$ совпадает с классом Никольского $H_{p}^{r}$. Как известно, подпространства тригонометрических полиномов со спектром из гиперболических крестов в ряде случаев оптимальны по порядку в смысле поперечников по Колмогорову для классов Никольского $H_{p}^{r}$ (см. [6]).

Tеорема 5 (многомерная теорема Джексона). Пусть функиия $f(\mathbf{x})=$ $f\left(x_{1}, \ldots, x_{d}\right)$ имеет период $2 \pi$ по каждой переменной. Пусть, далее, $f(\mathbf{x}) \in L_{p}$, 
$1 \leqslant p<\infty$, либо $f(x) \in C, p=\infty$. Тогда существует константас, не зависящая от $N$, такая, что

$$
\begin{aligned}
& E_{Q(N)}(f)_{p} \leqslant c \cdot \frac{1}{N}|\theta(N)|^{1 / p_{0}}, \quad 1 \leqslant p<\infty, \\
& E_{Q(N)}(f)_{\infty} \leqslant c \cdot \frac{1}{N}|\theta(N)|,
\end{aligned}
$$

где $p_{0}=\min \{p ; 2\},|\theta(N)|-$ количество әлементов множества $\theta(N)$.

Эта теорема вытекает из теоремы 4.

\section{Список литературы}

1. Руновский K. В. Об одной оценке для интегрального модуля гладкости // Изв. вузов. Сер. матем. 1992. № 1. С. 78-80.

2. Руновский K. B. О приближении семействами линейных полиномиальных операторов в пространствах $L_{p}, 0<p<1 / /$ Матем. сб. 1994. Т. 185. №8. С. 81-102.

3. Сторохенко Э.А., Кротов В.Г., Освальд П. Прямые и обратные теоремы типа Джексона в пространствах $L_{p}, 0<p<1 / /$ Матем. сб. 1975. Т. 98(140). С. 395-415.

4. Ульянов П. Л. Теоремы вложения и соотношения между наилучшими приближениями (модулями непрерывности) в разных метриках // Матем. сб. 1970. Т. 81. № 1. С. 104-131.

5. Пустовойтов H. H. Многомерная теорема Джексона в пространстве $L_{p} / /$ Матем. заметки. 1992. Т. 52. №1. С. 105-113.

6. Темляков В. Н. Приближение функций с ограниченной смешанной производной // Труды МИАН. 1986. Т. 178. С. 1-112.

7. Темляков $B . H$. О приближении функций нескольких переменных тригонометрическими полиномами с гармониками из гиперболических крестов // Укр. матем. журн. 1989. Т. 41. № 4. C. 518-524.

Московская государственная академия

Поступила в редакцию автомобилного и тракторного машиностроения

08.02 .1996 